\title{
THE FD-METHOD FOR SOLVING STURM-LIOUVILLE PROBLEMS WITH SPECIAL SINGULAR DIFFERENTIAL OPERATOR
}

\author{
V. L. MAKAROV, D. V. DRAGUNOV, AND YA. V. KLIMENKO
}

\begin{abstract}
A superexponentially convergent method for solving the SturmLiouville problem with Legendre's differential operator is given. The presented method (called the FD-method) is based on the coefficient approximation methods (CAM) and the homotopy approach. Sufficient convergence conditions of the proposed method are stated and rigorously substantiated. The algorithms for software implementation of the proposed method are described. The numerical examples included in the paper confirm the theoretical conclusions.
\end{abstract}

\section{INTRODUCTION}

There is a great range of numerical methods for solving eigenvalue problems for second-order differential operators (see [10]). Known numerical techniques for eigenvalue problems can be divided into two groups: methods based on direct approximation of solutions of the differential equation and methods based on approximation of the coefficients of the differential equation (see [8]).

The methods of the first group include the finite difference, finite elements, and spectral method, which are extensively treated through theoretical investigations as well as developed software. The main idea of this approach is to replace the eigenfunctions by piecewise polynomial functions. That results in an approximation of the differential equation with a system of linear algebraic equations. Due to the different nature of the original differential operator and the approximating algebraic operator the numerical solution approaches the exact solution only for lowindexed eigenvalues and the corresponding eigenfunctions. Hence, such numerical techniques are effective for finding low-indexed eigenvalues but not effective for finding high-indexed eigenvalues 8 .

The methods in the second group are based on the general idea of the coefficient approximation methods (hereinafter referred to as the CAM), also known as the Pruess methods. The idea is to replace the coefficient functions of the problem with a simpler approximation and then solve the approximating problem (see [10]). It was first proposed by Kryloff and Bogolioubov (1926) in [5] for piecewise constant approximation. S. Pruess provided a thorough convergence and error analysis of such methods when piecewise polynomial approximation is used. Except for Gordon, who uses linear functions, the earlier references all confine themselves to piecewise constant approximations, and this is the most practical choice; otherwise

Received by the editor August 1, 2011 and, in revised form, October 6, 2011.

2010 Mathematics Subject Classification. Primary 65L15, 65L20; Secondary 33D15, 68W99.

Key words and phrases. Singular Sturm-Liouville problem, FD-method, coefficient approximation methods, Legendre functions, Stenger's formula. 
the approximating problem may not be easier to solve numerically than the original. It is well known (see [10]) that if a piecewise constant approximation with the maximum step size $h$ is used, the error of the CAM is estimated by $O(h)$. Hence, a crucial problem arises when we use the CAM: to halve the error we have to double the computation time. This problem in many cases can be solved by using the functional-discrete method (hereinafter referred to as the FD-method) which is a substantial generalization of the CAM for solving Sturm-Liouville problems of different kinds. The algorithm of the FD-method was first described and justified for the linear Sturm-Liouville problem in [7. Generally speaking, it has two main steps: first, you have to find the initial approximation by applying the CAM; then, you have to calculate a sufficient number of corrections to get the required accuracy. The second step is provided by the homotopy method (also known as the perturbation method, the continuation method or the successive loading method; see, for example, [2]). Since the publication of paper [7] the application field of the FD-method has been substantially extended, especially towards the nonlinear Sturm-Liouville problems (see [3]).

In the present paper we consider the following singular Sturm-Liouville problem:

$$
\begin{gathered}
\frac{d}{d x}\left[\left(1-x^{2}\right) \frac{d u(x)}{d x}\right]-q(x) u(x)=\lambda u(x), \quad x \in(-1,1), \\
\lim _{x \rightarrow \pm 1}\left(1-x^{2}\right) \frac{d u(x)}{d x}=0 .
\end{gathered}
$$

We may encounter problems of this kind when solving partial differential equations via separation of variables as it was, for example, with the hydrogen-molecule ion's equation in [1] (see [11, pp. 167-170).

Since the problem (1.1), (1.2) has singularities at points \pm 1 , we cannot directly apply the algorithm of the FD-method proposed in [7]. However, a modified FDmethod technique for problems with such singularities was presented in [6]. Let us briefly state the algorithm of the FD-method described in [6].

According to the FD-method we approximate the eigenvalues and eigenfunctions of problem (1.1), (1.2) with the following truncated series:

$$
\stackrel{m}{\lambda}_{n}=\sum_{j=0}^{m} \lambda_{n}^{(j)}, \quad \stackrel{m}{u}_{n}(x)=\sum_{j=0}^{m} u_{n}^{(j)}(x),
$$

where $\lambda_{n}^{(j)}$ and $u_{n}^{(j)}(x), i=1,2, \ldots, m$ can be determined from the following recurrence problems:

$$
\begin{gathered}
\frac{d}{d x}\left[\left(1-x^{2}\right) \frac{d u_{n}^{(j)}(x)}{d x}\right]+\lambda_{n}^{(i)} u_{n}^{(j)}(x) \\
=-\sum_{s=0}^{j-1} \lambda_{n}^{(j-s)} u_{n}^{(s)}(x)+q(x) u_{n}^{(j-1)}(x)=F_{n}^{(j)}(x), \quad x \in(-1,1)
\end{gathered}
$$

with boundary conditions

$$
\lim _{x \rightarrow \pm 1}\left[\left(1-x^{2}\right) \frac{d u_{n}^{(j)}(x)}{d x}\right]=0,
$$

\footnotetext{
${ }^{1}$ The positive integer number $m$ is called the rank of FD-method.
} 
and orthogonality conditions

$$
\int_{-1}^{1} u_{n}^{(j)}(x) u_{n}^{(0)}(x) d x=0, \quad j=1,2, \ldots, m .
$$

The initial data $\lambda_{n}^{(0)}, u_{n}^{(0)}(x)$ needed to start the recurrence process can be found by solving the eigenvalue problem called the basic problem:

$$
\begin{gathered}
\frac{d}{d x}\left[\left(1-x^{2}\right) \frac{d u_{n}^{(0)}(x)}{d x}\right]+\lambda_{n}^{(0)} u_{n}^{(0)}(x)=0, \quad x \in(-1,1), \\
\lim _{x \rightarrow \pm 1}\left[\left(1-x^{2}\right) \frac{d u_{n}^{(0)}(x)}{d x}\right]=0 .
\end{gathered}
$$

The solutions to problem (1.7), (1.8) can be expressed in the form of

$$
\lambda_{n}^{(0)}=n(n+1), \quad u_{n}^{(0)}(x)=\sqrt{\frac{2 n+1}{2}} P_{n}(x),
$$

where $P_{n}(x)$ denotes the Legendre polynomial of the first kind of order $n \in \mathbb{N}$ (see [4]). The following statement about the convergence of the FD-method as described above was proved in [6].

Theorem 1.1. Let the function $q(x)$ belong to the class $Q^{0}[-1,1]$ of piecewise continuous functions defined on $[-1,1]$. Suppose that the positive integer $n_{0}$ satisfies the inequality

$$
q_{n_{0}}=\frac{2\|q(x)\|_{\infty,[-1,1]}}{n_{0}}<1 .
$$

Then the FD-method (1.4)-(1.9) has a superexponential convergence rate for all indices $n \geq n_{0}$ :

$$
\begin{gathered}
\left\|u_{n}(x)-\stackrel{m}{u}_{n}(x)\right\| \leq \frac{\left(q_{n}\right)^{m+1}}{1-q_{n}} \alpha_{m+1}, \quad\left|\lambda_{n}-\stackrel{m}{\lambda}_{n}\right| \leq \frac{\left(q_{n}\right)^{m}}{1-q_{n}}\|q(x)\|_{\infty} \alpha_{m}, \\
n=n_{0}, n_{0}+1, \ldots,
\end{gathered}
$$

where

$$
\alpha_{m+1}=2 \frac{(2 m-1) ! !}{(2 m+2) ! !} \leq \frac{1}{(m+1) \sqrt{\pi m}}, \quad\|q(x)\|_{\infty,[-1,1]}=\max _{x \in[-1,1]}|q(x)| .
$$

Theorem 1.1 was proved in [6] for the case when $q(x)=q(-x)$. However, using the same technique one can prove an analogous theorem for the case of an arbitrary function $q(x)$ from $Q^{0}[-1,1]$ without considerable difficulties.

If we try to apply the FD-method (1.4)-(1.9) to approximate the eigensolutions $\lambda_{n}, u_{n}(x)$ with $n<2\|q(x)\|_{\infty,[-1,1]}$ (see inequality (1.10) ) we might find it divergent. The reason for that is the lack of approximation for $q(x)$ in equation (1.7) of the basic problem. To overcome such difficulties we have to apply the general scheme of the FD-method using the approximation of the function $q(x)$ on $[-1,1]$ with a piecewise constant function $\bar{q}(x)$. 
2. The general Algorithm of the FD-Method For SOlving the STURM-LIOUVILLE PROBLEM WITH LEGENDRE DIFFERENTIAL OPERATOR:

\section{A THEORETICAL JUSTIFICATION}

To make the general algorithm of the FD-method easier to understand, let us consider the following general problem:

$$
\begin{gathered}
\frac{\partial}{\partial x}\left[\left(1-x^{2}\right) \frac{\partial u(x, t)}{\partial x}\right]+[\lambda(t)-\bar{q}(x)-t(q(x)-\bar{q}(x))] u(x, t)=0, \\
x \in(-1,1), t \in[0,1], \\
\lim _{x \rightarrow \pm 1}\left(1-x^{2}\right) \frac{\partial u(x, t)}{\partial x}=0, \quad \forall t \in[0,1] .
\end{gathered}
$$

To define the function $\bar{q}(x)$, we have to introduce a mesh on the interval $[-1,1]$ :

$$
\bar{\omega}=\left\{-1=x_{0}<x_{1}<\cdots<x_{N-1}<x_{N}=1\right\}, \quad h=\max _{i=1,2, \ldots, N}\left(x_{i}-x_{i-1}\right) .
$$

We also require that all points of discontinuity of the function $q(x)$ on the interval $[-1,1]$ be contained in the set $\bar{\omega}$.

One can choose $\bar{q}(x)$ in many possible ways. For example,

$$
\bar{q}(x)=\bar{q}(x, \bar{\omega})=q\left(\frac{1}{2}\left(x_{i-1}+x_{i}\right)\right), \quad x \in\left[x_{i-1}, x_{i}\right), \quad i=1,2, \ldots, N
$$

or

$$
\bar{q}(x)=\bar{q}(x, \bar{\omega})=\frac{1}{2}\left(q\left(x_{i-1}\right)+q\left(x_{i}\right)\right), \quad x \in\left[x_{i-1}, x_{i}\right), \quad i=1,2, \ldots, N,
$$

but in any case the piecewise constant function $\bar{q}(x, \bar{\omega})$ must have the following property:

$$
\lim _{h \rightarrow 0}\left(\max _{x \in[-1,1]}|\bar{q}(x, \bar{\omega})-q(x)|\right)=0 .
$$

It is easy to see that if we make $t=1$, then problem (2.1), (2.2) will be reduced to problem (1.1), (1.2). Assume that the solution to problem (2.1), (2.2) can be expressed in the form of a series:

$$
\lambda_{n}=\sum_{j=0}^{\infty} t^{(j)} \lambda_{n}^{(j)}, \quad u_{n}(x, t)=\sum_{j=0}^{\infty} t^{j} u_{n}^{(j)}(x)
$$

and

$$
\begin{gathered}
\frac{\partial u_{n}(x, t)}{\partial x}=\sum_{j=0}^{\infty} t^{j} \frac{\partial u_{n}^{(j)}(x)}{\partial x}, \quad \frac{\partial^{2} u_{n}(x, t)}{\partial x^{2}}=\sum_{j=0}^{\infty} t^{j} \frac{\partial^{2} u_{n}^{(j)}(x)}{\partial x^{2}} \\
\forall x \in(-1,1), \forall t \in[0,1] .
\end{gathered}
$$

The unknown summands of the series (2.5) are the solutions to the recurrence problems

$$
\begin{gathered}
\frac{d}{d x}\left[\left(1-x^{2}\right) \frac{d u_{n}^{(j)}(x)}{d x}\right]+\left[\lambda_{n}^{(0)}-\bar{q}(x)\right] u_{n}^{(j)}(x) \\
=-\sum_{s=0}^{j-1} \lambda_{n}^{(j-s)} u_{n}^{(s)}(x)+[q(x)-\bar{q}(x)] u_{n}^{(j-1)}(x) \equiv F_{n}^{(j)}(x), \quad x \in(-1,1),
\end{gathered}
$$


with the boundary conditions

$$
\lim _{x \rightarrow \pm 1}\left[\left(1-x^{2}\right) \frac{d u_{n}^{(j)}(x)}{d x}\right]=0
$$

matching conditions

$$
\left[u_{n}^{(j)}(x)\right]_{x=x_{i}}=0, \quad\left[\frac{d u_{n}^{(j)}(x)}{d x}\right]_{x=x_{i}}=0, i=1,2, \ldots, N-1,
$$

and an additional requirement that

$$
\begin{gathered}
\int_{-1}^{1} u_{n}^{(j)}(x) u_{n}^{(0)}(x) d x=\delta_{0, j}= \begin{cases}1, & \text { if } j=0, \\
0, & \text { if } j \neq 0,\end{cases} \\
j=0,1, \ldots, m .
\end{gathered}
$$

Here square brackets denote the jump of the function at the point $x=x_{i}$.

Out of problems (2.7)-(2.9) it is worth accentuating the first one $(j=0)$ :

$$
\begin{gathered}
\frac{d}{d x}\left[\left(1-x^{2}\right) \frac{d u_{n}^{(0)}(x)}{d x}\right]+\left[\lambda_{n}^{(0)}-\bar{q}(x)\right] u_{n}^{(0)}(x)=0, \quad x \in(-1,1), \\
\lim _{x \rightarrow \pm 1}\left[\left(1-x^{2}\right) \frac{d u_{n}^{(0)}(x)}{d x}\right]=0, \quad \int_{-1}^{1}\left(u_{n}^{(0)}(x)\right)^{2} d x=1, \\
{\left[u_{n}^{(0)}(x)\right]_{x=x_{i}}=0, \quad\left[\frac{d u_{n}^{(0)}(x)}{d x}\right]_{x=x_{i}}=0 .}
\end{gathered}
$$

As mentioned above, problem (2.11)-2.13) is called the basic problem. It is easy to verify that the differential operator

$$
L(\cdot)=\frac{d}{d x}\left[\left(1-x^{2}\right) \frac{d(\cdot)}{d x}\right]-\bar{q}(x)(\cdot)
$$

is self-adjoint in Sobolev space

$$
\bar{W}^{2,1}(-1,1)=\left\{f(x) \in W^{2,1}(-1,1): \lim _{x \rightarrow \pm 1}\left(1-x^{2}\right) f^{\prime}(x)=0\right\} .
$$

This fact implies that the eigenfunctions $u_{n}^{(0)}(x), n=0,1, \ldots$ of problem (2.11)(2.13) generate a complete orthogonal system in $\bar{W}^{2,1}(-1,1)$ and the corresponding eigenvalues $\lambda_{n}^{(0)},\left(\lambda_{i}^{(0)}<\lambda_{j}^{(0)}\right.$ when $\left.i<j, i, j=0,1,2 \ldots\right)$ are simple.

Suppose that the basic problem is solved and the eigenpair $\lambda_{n}^{(0)}, u_{n}^{(0)}(x)$ is one of its eigensolutions.

It is well known that problems (2.7)-(2.9) for $j=1,2, \ldots, m$ can be solved if and only if the following equality holds:

$$
\int_{-1}^{1} u_{n}^{(0)}(x) F_{n}^{(j)}(x) d x=0 .
$$


Combining equality (2.15) with (2.7) we get the formula for computing $\lambda_{n}^{(j)}$ :

$$
\lambda_{n}^{(j)}=-\sum_{s=1}^{j-1} \lambda_{n}^{(j-s)} \int_{-1}^{1} u_{n}^{(s)}(x) u_{n}^{(0)}(x) d x+\int_{-1}^{1}(q(x)-\bar{q}(x)) u_{n}^{(j-1)}(x) u_{n}^{(0)}(x) d x .
$$

Taking into account (2.10) we can simplify formula (2.16) as follows:

$$
\lambda_{n}^{(j)}=\int_{-1}^{1}(q(x)-\bar{q}(x)) u_{n}^{(j-1)}(x) u_{n}^{(0)}(x) d x .
$$

One can easily verify that the function $u_{n}^{(j)}(x)$ can be represented in the form of the series

$$
u_{n}^{(j)}(x)=\sum_{\substack{p=0 \\ p \neq n}}^{\infty} \frac{\int_{-1}^{1} F_{n}^{(j)}(x) u_{p}^{(0)}(x) d x}{\lambda_{n}^{(0)}-\lambda_{p}^{(0)}} u_{p}^{(0)}(x) .
$$

Formulas (2.17) and (2.18) yield estimations

$$
\left|\lambda_{n}^{(j)}\right| \leq\|q(x)-\bar{q}(x)\|_{\infty,[-1,1]}\left\|u_{n}^{(j-1)}(x)\right\|
$$

and

$$
\begin{gathered}
\left\|u_{n}^{(j)}(x)\right\| \leq M_{n}\left\|F_{n}^{(j)}(x)\right\| \\
=M_{n}\left\{\left\|\sum_{s=1}^{j-1} \lambda_{n}^{j-s} u_{n}^{(s)}(x)-(q(x)-\bar{q}(x)) u_{n}^{(j-1)}(x)\right\|^{2}-\left(\lambda^{j+1}\right)^{2}\right\}^{\frac{1}{2}} \\
\leq M_{n}\left\{\sum_{s=1}^{j-1}\left|\lambda_{n}^{(j-s)}\right|\left\|u_{n}^{(s)}(x)\right\|+\|q(x)-\bar{q}(x)\|_{\infty,[-1,1]}\left\|u_{n}^{(j-1)}(x)\right\|\right\} \\
\leq M_{n}\|q(x)-\bar{q}(x)\|_{\infty,[-1,1]} \sum_{s=0}^{j-1}\left\|u_{n}^{(j-1-s)}\right\|\left\|u_{n}^{(s)}(x)\right\|,
\end{gathered}
$$

respectively, where

$$
\begin{gathered}
M_{n}=\max \left\{\left(\lambda_{n}^{(0)}-\lambda_{n-1}^{(0)}\right)^{-1},\left(\lambda_{n+1}^{(0)}-\lambda_{n}^{(0)}\right)^{-1}\right\}, \quad n=1,2, \ldots \\
M_{0}=\left(\lambda_{1}^{(0)}-\lambda_{0}^{(0)}\right)^{-1}
\end{gathered}
$$

and $\|\cdot\|$ is the common $L_{2}$-norm, $\|f(x)\|=\sqrt{\int_{-1}^{1} f^{2}(x) d x}$.

Multiplying both sides of inequality (2.20) by $\left(M_{n}\|q(x)-\bar{q}(x)\|_{\infty,[-1,1]}\right)^{-j}$ and using estimation (2.19) we obtain

$$
\frac{\left\|u_{n}^{(j)}(x)\right\|}{\left(M_{n}\|q(x)-\bar{q}(x)\|_{\infty,[-1,1]}\right)^{j}} \leq \sum_{s=0}^{j-1} \frac{\left\|u_{n}^{(j-s-1)}(x)\right\|\left\|u_{n}^{(s)}(x)\right\|}{\left(M_{n}\|q(x)-\bar{q}(x)\|_{\infty,[-1,1]}\right)^{j-1}} .
$$


Using the notation

$$
v_{j}=\left\|u_{n}^{(j)}(x)\right\|\left(M_{n}\|q(x)-\bar{q}(x)\|_{\infty,[-1,1]}\right)^{-j}=\left\|u_{n}^{(j)}(x)\right\| \bar{r}_{n}^{-j},
$$

we can represent inequality (2.22) as

$$
v_{j} \leq \sum_{s=0}^{j-1} v_{j-s-1} v_{s}, j=1,2, \ldots, v_{0}=\left\|u_{n}^{(0)}(x)\right\|=1 .
$$

It is easy to see that the sequence $\left\{V_{i}\right\}$ defined by the recurrence formula

$$
V_{j}=\sum_{s=0}^{j-1} V_{j-1-s} V_{s}, \quad V_{0}=1, \quad j=0,1, \ldots,
$$

satisfies inequalities

$$
V_{j} \geq v_{j}, \quad j=0,1, \ldots
$$

Let us consider the generating function $f(z)$ defined by the formula

$$
f(z)=\sum_{s=0}^{\infty} V_{s} z^{s}, \quad z \in \mathbb{R} .
$$

From (2.25) we obtain the equation with respect to $f(z)$ :

$$
f(z)=z f^{2}(z)+1, \quad z \in(-\rho, \rho)
$$

where $\rho$ denotes the convergence radius for the power series (2.27). The solution to equation (2.28) that satisfies the condition $f(0)=1$ is

$$
f(z)=(2 z)^{-1}(1-\sqrt{1-4 z})=\left(2 z+\sum_{s=2}^{\infty} \frac{(2 s-3) ! !}{(2 s) ! !}(4 z)^{s}\right)(2 z)^{-1}, \quad z \in\left[-\frac{1}{4}, \frac{1}{4}\right]
$$

and

$$
V_{j}=2 \frac{(2 j-1) ! !}{(2 j+2) ! !} 4^{j}=\alpha_{j} 4^{j}, \quad j=1,2, \ldots, \quad(-1) ! ! \stackrel{\text { def }}{=} 1 .
$$

As follows from (2.29), the convergence radius of the power series (2.27) is equal to $\frac{1}{4}$ and, additionally, the series converges at the points $z= \pm \frac{1}{4}$.

Going back to formula (2.23) and using inequalities (2.26) along with formula (2.30) we get the estimation

$$
\left\|u_{n}^{(j)}(x)\right\|=\left(M_{n}\|q(x)-\bar{q}(x)\|_{\infty,[-1,1]}\right)^{j} v_{j} \leq\left(4 \bar{r}_{n}\right)^{j} \alpha_{j}=r_{n}^{j} \alpha_{j}
$$

and inequality (2.31) gives us the following estimations of the FD-method's convergence rate:

$$
\begin{aligned}
& \left\|u_{n}(x)-\stackrel{m}{u}_{n}(x)\right\| \leq\left\{\begin{array}{ccc}
\frac{r_{n}^{m+1}}{1-r_{n}} \alpha_{m+1}, & \text { if } & r_{n}<1, \\
\sum_{j=m+1}^{\infty} \frac{1}{(j+1) \sqrt{\pi j}}, & \text { if } & r_{n}=1
\end{array}\right. \\
& \left|\lambda_{n}-\stackrel{m}{\lambda}_{n}\right| \leq\left\{\begin{array}{cc}
\|q(x)-\bar{q}(x)\|_{\infty,[-1,1]} \frac{r_{n}^{m}}{1-r_{n}} \alpha_{m}, & \text { if } \quad r_{n}<1, \\
\|q(x)-\bar{q}(x)\|_{\infty,[-1,1]} \sum_{j=m}^{\infty} \frac{1}{(j+1) \sqrt{\pi j},}, & \text { if } \quad r_{n}=1 .
\end{array}\right.
\end{aligned}
$$


The infinite sum in the right sides of inequalities (2.32) and (2.33) can be easily estimated as

$$
\sum_{j=m}^{\infty} \frac{1}{(j+1) \sqrt{\pi j}} \leq \int_{m-1}^{\infty} \frac{1}{(x+1) \sqrt{x \pi}} d x=\frac{2}{\sqrt{\pi}} \arctan \left(\frac{1}{\sqrt{m+1}}\right) .
$$

Hereby, we have proved the following theorem.

Theorem 2.1. Assume that $q(x)$ belongs to $Q^{0}[-1,1]$ and the constant $\bar{r}_{n}=$ $M_{n}\|q(x)-\bar{q}(x)\|_{\infty,[-1,1]}$ satisfies the inequality $\bar{r}_{n} \leq \frac{1}{4}$. Under this assumption the FD-method (2.5), (2.7) -(2.10) converges faster than a geometric series with denominator $r_{n}=4 \bar{r}_{n}$ (which is to say, superexponentially). The estimations of its convergence rate are given by formulas (2.32), (2.33).

Before passing on to the next section let us investigate the question of asymptotical behavior of the parameter $M_{n}$ in (2.21) as $n$ tends to $+\infty$. It is easy to see that the basic problem (2.11) -(2.13) is equivalent to the auxiliary eigenvalue problem

$$
\frac{\partial}{\partial x}\left[\left(1-x^{2}\right) \frac{\partial v(x, \tau)}{\partial x}\right]+[\mu(\tau)-\tau \bar{q}(x)] v(x, \tau)=0, \quad x \in(-1,1), \tau \in[0,1],
$$

$$
\begin{gathered}
\lim _{x \rightarrow \pm 1}\left[\left(1-x^{2}\right) \frac{\partial v(x, \tau)}{\partial x}\right]=0, \quad \int_{-1}^{1}(v(x, \tau))^{2} d x=1, \forall \tau \in[0,1], \\
{[v(x, \tau)]_{x=x_{i}}=0, \quad\left[\frac{\partial v(x, \tau)}{\partial x}\right]_{x=x_{i}}=0, \forall \tau \in[0,1], i=1,2, \ldots, N-1,}
\end{gathered}
$$

when $\tau=1$. On the other hand, from the proof of Theorem 1.1] (see 6]) it follows that for a sufficiently large number $n$, namely $2^{2}$

$$
n \geq n_{0}=\left\{\begin{array}{cll}
{\left[2\|q(x)\|_{\infty,[-1,1]}\right]+1} & \text { if } & \|q(x)\|_{\infty,[-1,1]} \geq \frac{1}{2}, \\
0 & \text { if } & \|q(x)\|_{\infty,[-1,1]}<\frac{1}{2},
\end{array}\right.
$$

the eigensolution $\mu_{n}(\tau), v_{n}(x, \tau)$ to problem (2.35) 2.37) exists and can be expanded into a power series with respect to $\tau$ on the interval $[0,1]$ for all $x \in(-1,1)$; furthermore,

$$
\mu_{n}(0)=n(n+1), \mu_{n}(1)=\lambda_{n}^{(0)} .
$$

Hence, from equality (2.35) it is easy to obtain the equality

$$
\begin{gathered}
\frac{\partial}{\partial x}\left[\left(1-x^{2}\right) \frac{\partial^{2} v_{n}(x, \tau)}{\partial x \partial \tau}\right]+\left[\mu_{n}(\tau)-\tau \bar{q}(x)\right] \frac{\partial}{\partial \tau} v_{n}(x, \tau)=\left[\mu_{n}^{\prime}(\tau)-\bar{q}(x)\right] v_{n}(x, \tau) \\
x \in(-1,1), \tau \in[0,1]
\end{gathered}
$$

Then, applying the integral operator $\int_{-1}^{1} v_{n}(x, \tau)(\cdot) d x$, to both sides of equality (2.40) we derive that

$$
\mu_{n}^{\prime}(\tau)=\int_{-1}^{1}\left(v_{n}(x, \tau)\right)^{2} \bar{q}(x) d x, \quad \tau \in[0,1]
$$

\footnotetext{
${ }^{2}$ The square brackets in formula (2.38) denote the integer part function.
} 
Taking into account (2.39) and equality (2.41) we obtain the equality

$$
\lambda_{n}^{(0)}=n(n+1)+\int_{0}^{1} \int_{-1}^{1}\left(v_{n}(x, \tau)\right)^{2} \bar{q}(x) d x d \tau .
$$

Finally, equality (2.42) yields the following estimation:

$$
\lambda_{n+1}^{(0)}-\lambda_{n}^{(0)} \geq 2(n+1)-2\|q(x)\|_{\infty,[-1,1]}>0 .
$$

Hence, for a sufficiently large $n$ (see (2.38) $)$ we have arrived at an estimation that describes the asymptotical behavior of $M_{n}$ :

$$
M_{n} \leq\left(2 n-2\|q(x)\|_{\infty,[-1,1]}\right)^{-1} .
$$

Taking into account (2.38) and using formula (2.42) we can easily prove that if

$$
0 \leq q(x)<\frac{1}{2}, \quad \forall x \in[-1,1]
$$

then inequality (2.44) can be reduced as follows:

$$
M_{n}<(2 n-1)^{-1}, n=1,2, \ldots, \quad M_{0}<1 .
$$

Furthermore, assumption (2.45) provides the inequalities

$$
\lambda_{n+1}^{(0)}-\lambda_{n}^{(0)}>\lambda_{n}^{(0)}-\lambda_{n-1}^{(0)}, \quad n=1,2, \ldots,
$$

which allow us to simplify formula (2.21):

$$
M_{n}=\left(\lambda_{n}^{(0)}-\lambda_{n-1}^{(0)}\right)^{-1}<1, \quad n=1,2, \ldots, \quad M_{0}=M_{1} .
$$

3. The general Algorithm of the FD-Method: SOFTWARE IMPLEMENTATION

In this section we discuss the question of implementing the algorithm of the FD-method in detail.

The solution to the basic problem (2.11)-(2.13) can be represented in the form

$$
u_{n}^{(0)}(x)=A_{i} P_{\nu_{i}}(x)+B_{i} Q_{\nu_{i}}(x), \quad x \in\left[x_{i-1}, x_{i}\right], \quad A_{i}, B_{i} \in \mathbb{R}, \quad i=\overline{1, N},
$$

where

$$
\nu_{i}=\nu_{i}\left(\lambda_{n}^{(0)}\right)=\frac{1}{2}\left(-1 \pm \sqrt{1+4\left(\lambda_{n}^{(0)}-\bar{q}_{i}\right)}\right)^{3}, \quad \bar{q}_{i} \equiv \bar{q}(x), x \in\left[x_{i-1}, x_{i}\right)
$$

and $Q_{\nu_{i}}(x)$ denotes a Legendre function of the second kind (see [4]). It is well known that (see, for example, 4])

$$
\lim _{x \rightarrow-1}\left(1-x^{2}\right) \frac{d P_{\nu}(x)}{d x}=\frac{2 \sin (\pi \nu)}{\pi}, \quad \lim _{x \rightarrow 1}\left(1-x^{2}\right) \frac{d P_{\nu}(x)}{d x}=0,
$$

$$
\lim _{x \rightarrow-1}\left(1-x^{2}\right) \frac{d Q_{\nu}(x)}{d x}=\cos (\pi \nu), \quad \lim _{x \rightarrow 1}\left(1-x^{2}\right) \frac{d Q_{\nu}(x)}{d x}=1 .
$$

To satisfy boundary condition (2.12) we have to require that

$$
A_{1} \frac{2 \sin \left(\pi \nu_{1}\right)}{\pi}+B_{1} \cos \left(\pi \nu_{1}\right)=0, \quad B_{N}=0 .
$$

\footnotetext{
${ }^{3}$ Either "+" or "-" can be chosen.
} 
The constants $A_{i}, B_{i}$ for $i<N$ have to be found using matching conditions (2.13). This means that they can be found as solutions to the following recurrence systems of linear algebraic equations:

$$
\left\{\begin{aligned}
A_{i-1} P_{\nu_{i-1}}\left(x_{i-1}\right)+B_{i-1} Q_{\nu_{i-1}}\left(x_{i-1}\right) & =A_{i} P_{\nu_{i}}\left(x_{i-1}\right)+B_{i} Q_{\nu_{i}}\left(x_{i-1}\right) \\
A_{i-1} P_{\nu_{i-1}}^{\prime}\left(x_{i-1}\right)+B_{i-1} Q_{\nu_{i-1}}^{\prime}\left(x_{i-1}\right) & =A_{i} P_{\nu_{i}}^{\prime}\left(x_{i-1}\right)+B_{i} Q_{\nu_{i}}^{\prime}\left(x_{i-1}\right)
\end{aligned}\right.
$$

$i=2,3, \ldots, N$.

Let us consider the function

$$
\Phi(\lambda)=A_{1} \frac{2 \sin \left(\pi \nu_{1}\right)}{\pi}+B_{1} \cos \left(\pi \nu_{1}\right), \quad \nu_{1}=\nu_{1}(\lambda)
$$

where constants $A_{1}, B_{1}$ are derived from the following recurrence formulas (see (3.5) :

$$
\begin{gathered}
A_{i-1}=\frac{1}{\delta_{i-1}}\left(C_{i} Q_{\nu_{i-1}}^{\prime}\left(x_{i-1}\right)-D_{i} Q_{\nu_{i-1}}\left(x_{i-1}\right)\right), \\
B_{i-1}=\frac{1}{\delta_{i-1}}\left(D_{i} P_{\nu_{i-1}}\left(x_{i-1}\right)-C_{i} P_{\nu_{i-1}}^{\prime}\left(x_{i-1}\right)\right), \\
\delta_{i-1}=P_{\nu_{i-1}}\left(x_{i-1}\right) Q_{\nu_{i-1}}^{\prime}\left(x_{i-1}\right)-P_{\nu_{i-1}}^{\prime}\left(x_{i-1}\right) Q_{\nu_{i-1}}\left(x_{i-1}\right), \\
C_{i}=A_{i} P_{\nu_{i}}\left(x_{i-1}\right)+B_{i} Q_{\nu_{i}}\left(x_{i-1}\right), \\
D_{i}=A_{i} P_{\nu_{i}}^{\prime}\left(x_{i-1}\right)+B_{i} Q_{\nu_{i}}^{\prime}\left(x_{i-1}\right), \quad i=N, N-1, \ldots, 2
\end{gathered}
$$

with the initial data

$$
A_{N}=1, \quad B_{N}=0,
$$

and the values $\nu_{i}=\nu_{i}(\lambda)$ are defined by formula (3.2). It is easy to see that the eigenvalues of the basic problem (2.11) (2.13) coincide with the solutions of the equation

$$
\Phi(\lambda)=0
$$

Since the eigenvalues of the basic problem are simple, they can be found with arbitrary precision as the solutions to equation (3.8) using the bisection method described in Algorithm 1 below. It is worth emphasizing that in practice the value $\nu_{i}$, obtained from formula (3.2) can be complex. Therefore, the value of $\Phi(\lambda)$ computed using formulas (3.6) can be complex too. However, to avoid unnecessary difficulties related to using complex numbers in computation it is sufficient to take real parts of $P_{\nu_{i}}\left(x_{i}\right), P_{\nu_{i}}^{\prime}\left(x_{i}\right), Q_{\nu_{i}}\left(x_{i}\right), Q_{\nu_{i}}\left(x_{i}\right)$ only. This was done in the algorithm for the function ComputePhi described below.

Let $\lambda_{n}^{(0)}$ be a single fixed solution to equation (3.8). Using the coefficients $A_{i}, B_{i}$, $i=1,2, \ldots, N$, computed from formulas (3.6) with $\lambda=\lambda_{n}^{(0)}$ (see comments at the end of Algorithm 1) we can compute the corresponding eigenfunction $u_{n}^{(0)}(x)$ using formula (3.1).

To compute $\lambda_{n}^{(j)}$ we can use the formula

$$
\lambda_{n}^{(j)}=\left[\int_{-1}^{1}\left(u_{n}^{(0)}(x)\right)^{2} d x\right]^{-1} \int_{-1}^{1}(q(x)-\bar{q}(x)) u_{n}^{(j-1)}(x) u_{n}^{(0)}(x) d x .
$$




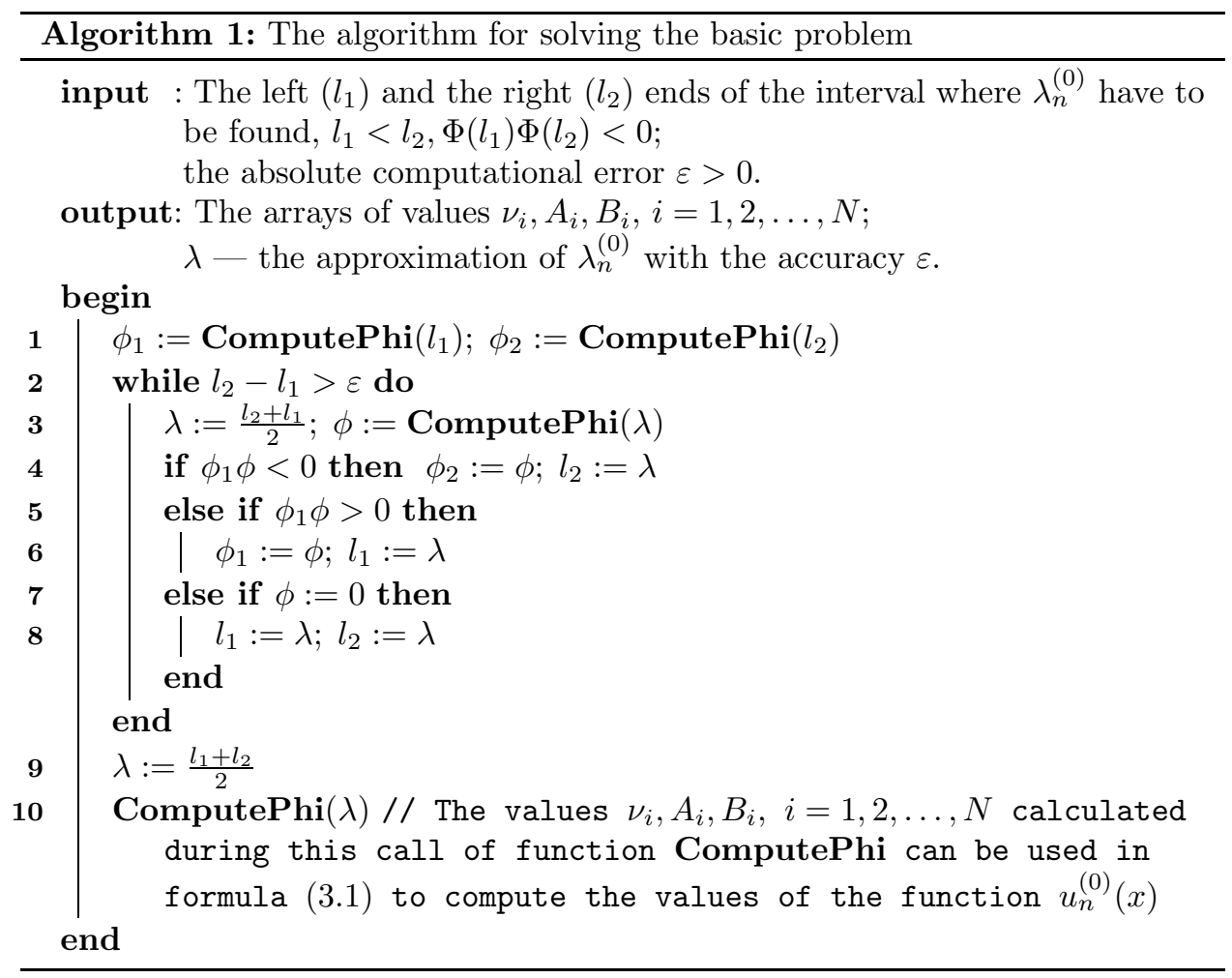

To compute the functions $u_{n}^{(j)}(x), j=1,2, \ldots, m$, it is convenient to use the formula

$$
\begin{gathered}
u_{n}^{(j)}(x)=E_{j} u_{n}^{(0)}(x)+\int_{-1}^{x} K(x, \xi) F_{n}^{(j)}(\xi) d \xi \\
=E_{j} u_{n}^{(0)}(x)+w_{n}(x) \int_{-1}^{x} u_{n}^{(0)}(\xi) F_{n}^{(j)}(\xi) d \xi-u_{n}^{(0)}(x) \int_{-1}^{x} w_{n}(\xi) F_{n}^{(j)}(\xi) d \xi,
\end{gathered}
$$

where

$$
\begin{gathered}
K(x, \xi)=w_{n}(x) u_{n}^{(0)}(\xi)-w_{n}(\xi) u_{n}^{(0)}(x) \\
E_{j}=-\left[\int_{-1}^{1}\left(u_{n}^{(0)}(x)\right)^{2} d x\right]^{-1} \int_{-1}^{1}\left\{u_{n}^{(0)}(x) \int_{-1}^{x} K(x, \xi) F_{n}^{(j)}(\xi) d \xi\right\} d x
\end{gathered}
$$

and $w_{n}(x)$ denotes a function obtained from the formula

$$
w_{n}(x)=A_{i} P_{\nu_{i}}(x)+B_{i} Q_{\nu_{i}}(x), \quad x \in\left[x_{i-1}, x_{i}\right], \quad i=\overline{1, N} .
$$

Here $A_{i}, B_{i}, i=1,2, \ldots, N$ can be computed recursively from formulas (3.6) with the initial data

$$
A_{N}=0, B_{N}=1
$$

and $\nu_{i}$ are computed by formula (3.2). 


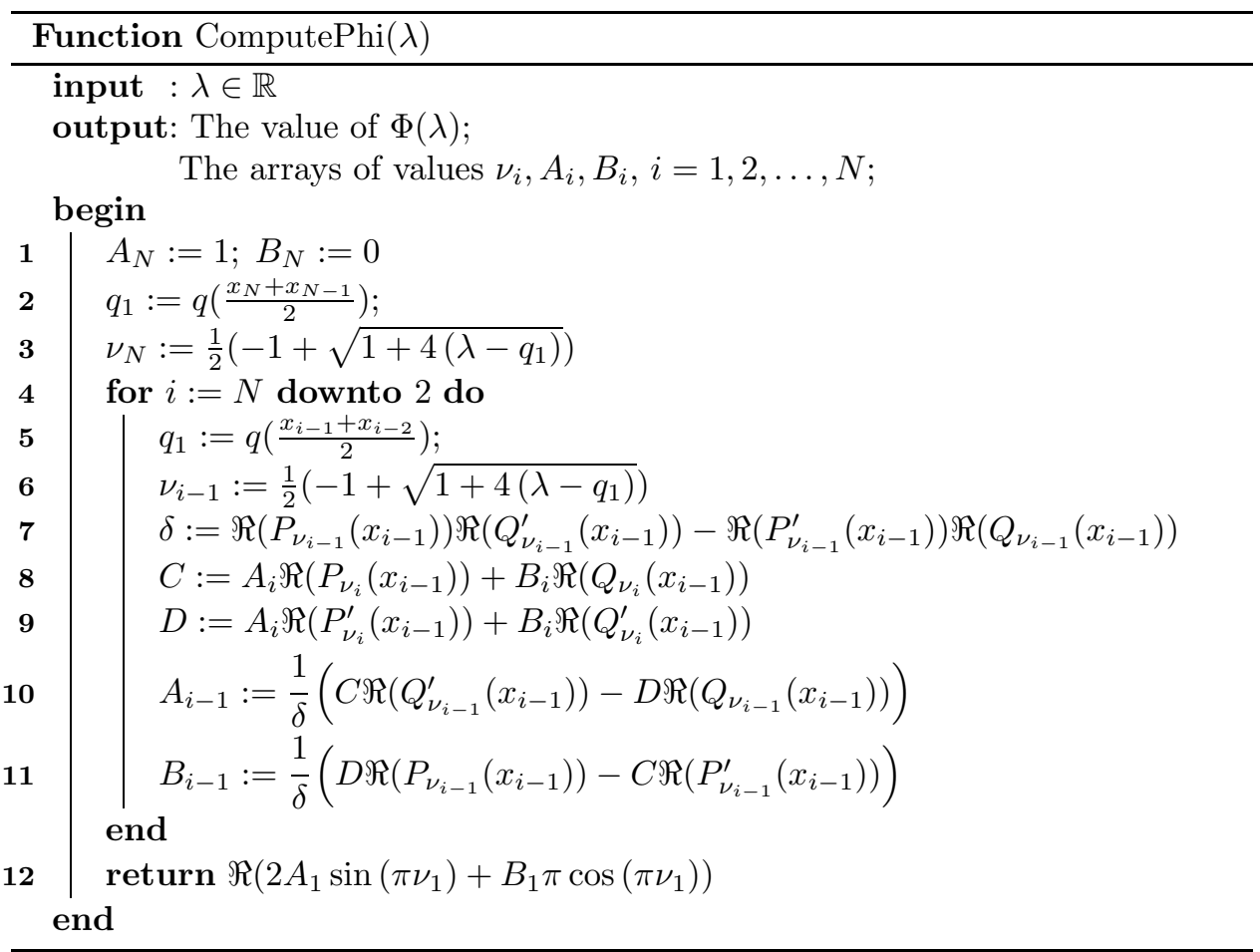

Taking into account the properties of Legendre functions (see [4]) it is easy to verify that

$$
\left.\frac{\partial K(x, \xi)}{\partial x}\right|_{\xi=x}=\left(1-x^{2}\right)^{-1}, \quad x \in(-1,1)
$$

and $K(x, \xi)$ has the properties of a Cauchy operator for inhomogeneous equations (2.7).

Generally speaking, the integrals in formulas (3.9) and (3.10) may not be analytically obtainable. Moreover, the integrands in these formulas are unbounded at the points \pm 1 . To compute these integrals it is convenient to use numerical methods. For example, the tanh rule (see [9]) and Stenger's formula (see [12]):

$$
\begin{aligned}
\int_{a}^{b} f(x) d x & \int_{-\infty}^{+\infty} f\left(\frac{a+b e^{\omega}}{1+e^{\omega}}\right) \frac{(b-a) d \omega}{\left(e^{-\omega / 2}+e^{\omega / 2}\right)^{2}} \\
\approx & h_{\operatorname{sinc}} \sum_{l=-K}^{K} f\left(\frac{a+b e^{l h_{\text {sinc }}}}{1+e^{l h_{\text {sinc }}}}\right) \frac{(b-a)}{\left(e^{-l h_{\text {sinc }} / 2}+e^{l h_{\text {sinc }} / 2}\right)^{2}}
\end{aligned}
$$

and

$$
\int_{a}^{z_{k}} f(x) d x \approx h_{\text {sinc }} \sum_{l=-K}^{K} \delta_{k-l}^{(-1)} f\left(\frac{a+b e^{l h_{\text {sinc }}}}{1+e^{l h_{\text {sinc }}}}\right) \frac{(b-a)}{\left(e^{-l h_{\text {sinc }} / 2}+e^{l h_{\text {sinc }} / 2}\right)^{2}} \sqrt[4]{4}
$$

${ }^{4}$ The function $f(x)$ needs to be sufficiently smooth on $(\mathrm{a}, \mathrm{b})$; see 12 . 
where

$z_{k}=\frac{a+b e^{h_{\text {sinc }} k}}{1+e^{h_{\text {sinc }} k}}, k=-K, \ldots, K, \quad \delta_{k}^{(-1)}=\frac{1}{2}+\int_{0}^{k} \frac{\sin (\pi t)}{\pi t} d t, k=-2 K, \ldots, 2 K$,

and $h_{\text {sinc }}=\sqrt{\frac{2 \pi}{K}}$ 占

Henceforth we require that the function $q(x)$ be analytical on every interval $\left(x_{i-1}, x_{i}\right), i=1,2, \ldots, N$. Before we can describe the algorithm we need to introduce some auxiliary notation:

$$
\begin{gathered}
z_{i, j}=\frac{x_{i-1}+x_{i} e^{h_{\text {sinc }} j}}{1+e^{h_{\text {sinc }} j}}, \quad \mu_{i, j}=\frac{\left(x_{i-1}-x_{i}\right)}{\left(e^{-j h_{\text {sinc }} / 2}+e^{j h_{\text {sinc }} / 2}\right)^{2}}, \\
\nu_{n}=\left[\int_{-1}^{1}\left(u_{n}^{(0)}(x)\right)^{2} d x\right]^{-1}, \quad i=1,2, \ldots, N, j=-K, \ldots, K .
\end{gathered}
$$

One possible algorithm for computing $\lambda_{n}^{(k)}$ and $u_{n}^{(k)}(x)$ for $k=1,2, \ldots, r, i=$ $1,2, \ldots, N, j=-K, \ldots, K$ is described below.

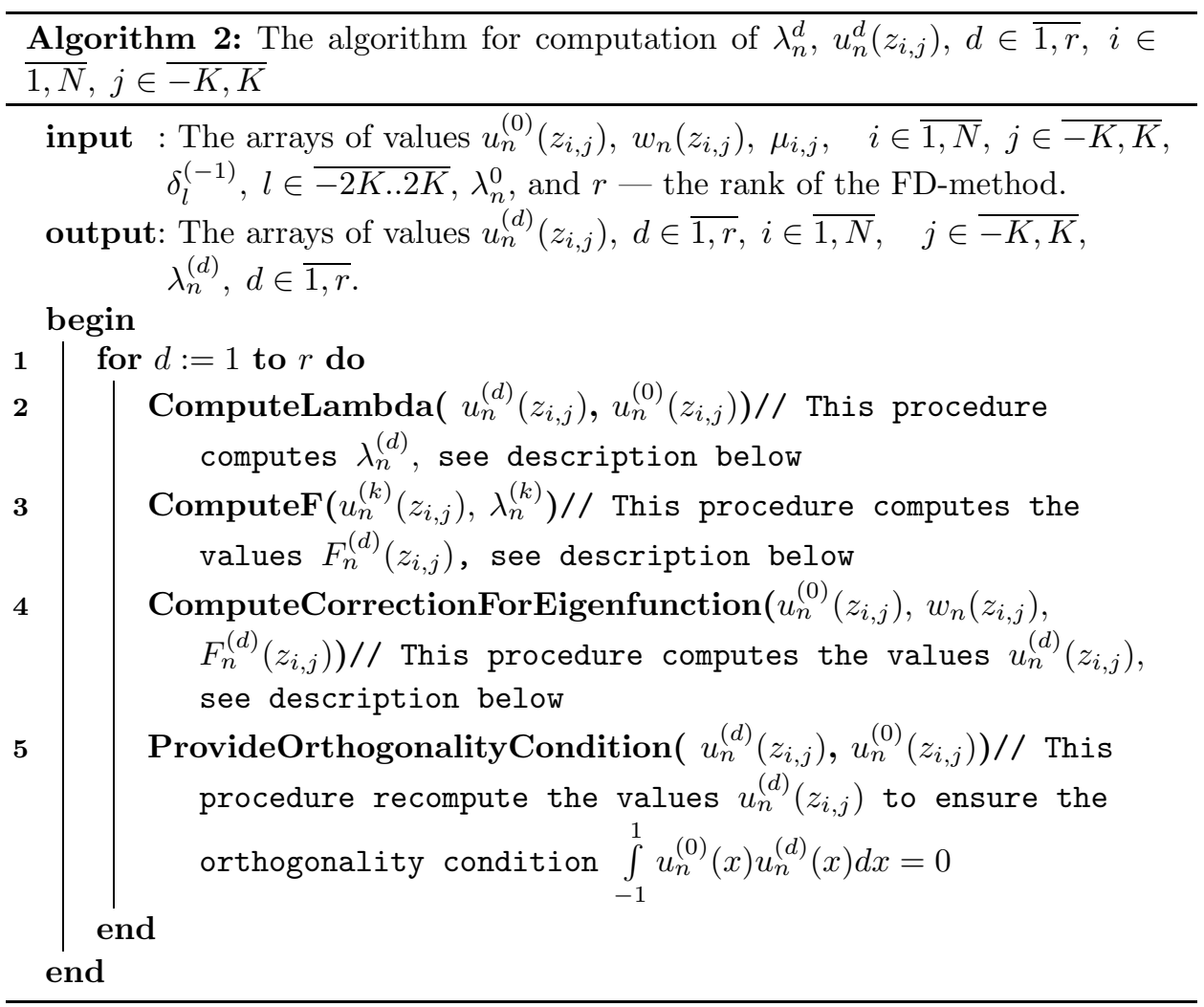

${ }^{5}$ For discussion on the optimal choice of value for $h_{\text {sinc }}$ see 12 . 

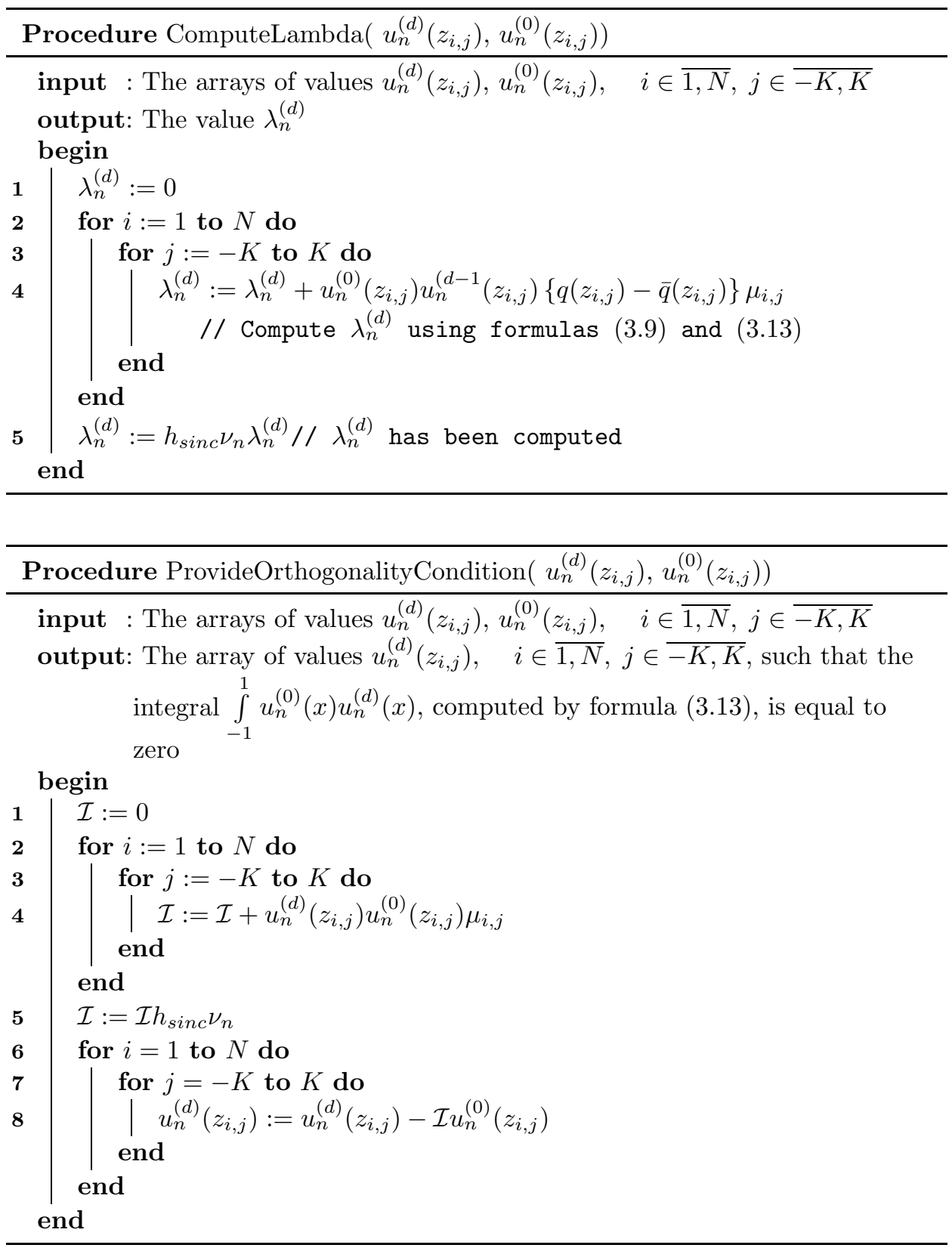

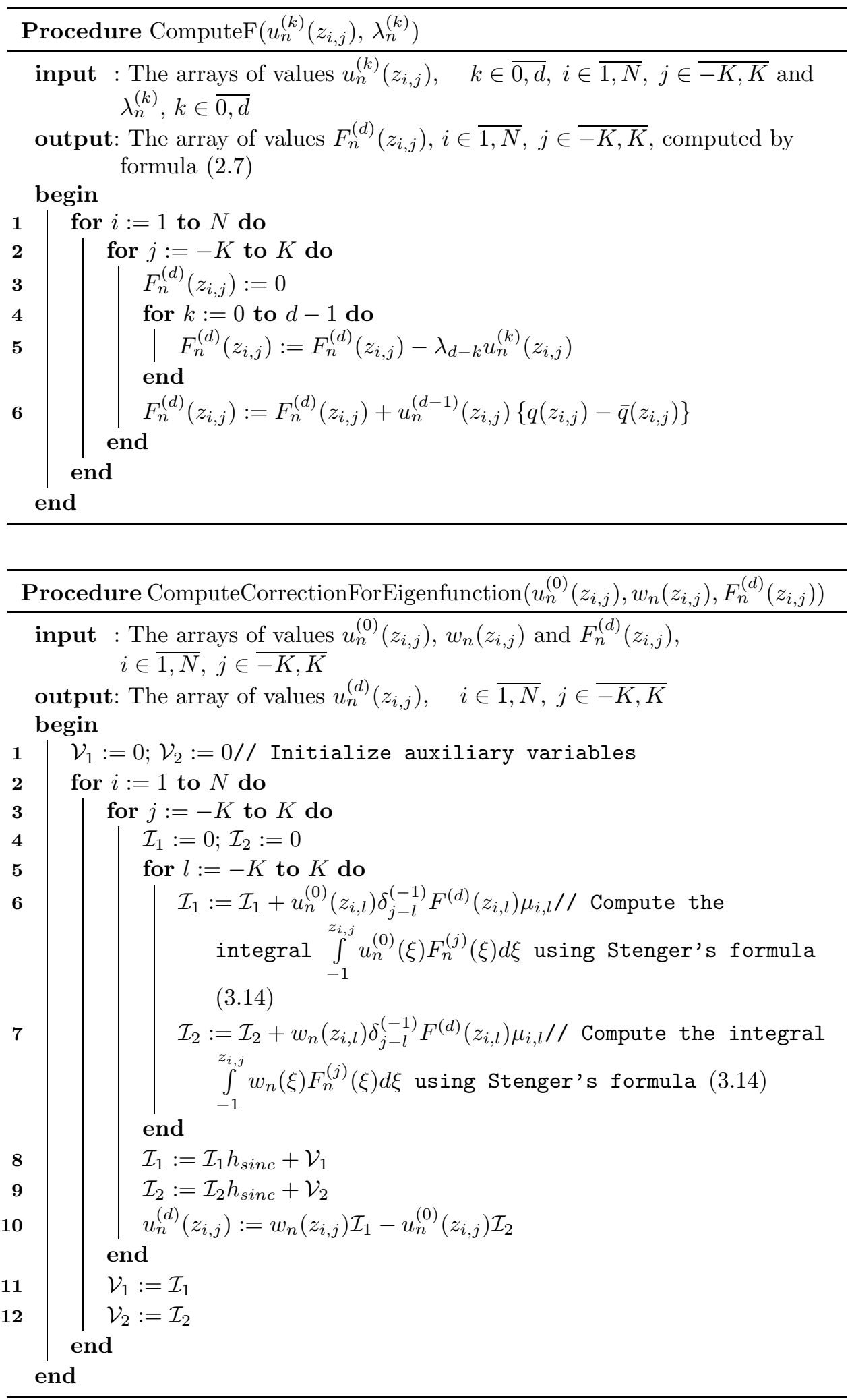
The algorithm described above is not the most efficient one. Nevertheless, the numerical results presented in the next section were successfully obtained using this algorithm. It is evident that the highest accuracy that can be achieved by increasing the rank $r$ of the FD-method is limited by the accuracy of the quadrature formulas (3.13), (3.14) used in the algorithm. The optimal choice of the parameters $r$ and $K$ remains a current issue. In the next section we will present three numerical examples. In the first example we apply quadrature formulas (3.13), (3.14) with $K=500$ and in the following two we use $K=350$.

\section{Numerical EXAMPLES}

Example 1. Let us consider the following Sturm-Liouville problem:

$$
\begin{gathered}
L(u(x))=\frac{d}{d x}\left[\left(1-x^{2}\right) \frac{d u(x)}{d x}\right]+(\lambda-q(x)) u(x)=0, \quad x \in(-1,1), \\
\lim _{x \rightarrow \pm 1}\left[\left(1-x^{2}\right) \frac{d u(x)}{d x}\right]=0
\end{gathered}
$$

with

$$
q(x)=x .
$$

Using the FD-method described in [6] and the computer algebra system Maple we have found that

$$
\begin{array}{lll}
\lambda_{0}^{(0)}=0, & u_{0}^{(0)}(x)=\frac{\sqrt{2}}{2}, \\
\lambda_{0}^{(1)}=0, & u_{0}^{(1)}(x)=-\frac{\sqrt{2} x}{4}, \\
\lambda_{0}^{(2)}=-\frac{1}{6}, & u_{0}^{(2)}(x)=\frac{\sqrt{2} x^{2}}{24}-\frac{\sqrt{2}}{72}, \\
\lambda_{0}^{(3)}=0, & u_{0}^{(3)}(x)=-\frac{\sqrt{2} x^{3}}{288}+\frac{5 \sqrt{2} x}{288}, \\
\lambda_{0}^{(4)}=\frac{11}{1080}, & u_{0}^{(4)}(x)=\frac{\sqrt{2} x^{4}}{5760}-\frac{\sqrt{2} x^{2}}{270}+\frac{311 \sqrt{2}}{259200}, \\
\lambda_{0}^{(5)}=0, & u_{0}^{(5)}(x)=-\frac{\sqrt{2} x^{3}}{172800}+\frac{1181 \sqrt{2} x}{2880}-\frac{\sqrt{2} x^{6}}{518400}, \\
\lambda_{0}^{(6)}=-\frac{47}{34020}, & u_{0}^{(6)}(x)=\frac{11237 \sqrt{2} x^{2}}{7257600}-\frac{76967 \sqrt{2}}{53760}+\frac{71772800}{457228800} .
\end{array}
$$

To control the accuracy of the results obtained by the FD-method we use the following functionals:

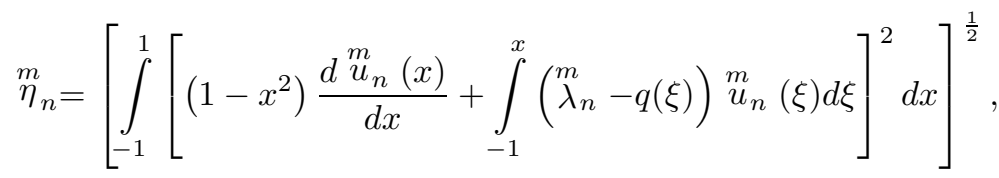

$$
\begin{aligned}
& \bar{\eta}_{n}=\left[\int_{-1}^{1}\left[\frac{d}{d x}\left[\left(1-x^{2}\right) \frac{d \stackrel{m}{u}_{n}(x)}{d x}\right]+\left(\stackrel{m}{\lambda_{n}}-q(x)\right) \stackrel{m}{u}_{n}(x)\right]^{2} d x\right]^{\frac{1}{2}} .
\end{aligned}
$$

We also compare the results of the FD-method with eigenvalues obtained by SLEIGN2 (see [1]). 
TABle 1. Example 1. The data obtained by SLEIGN2

\begin{tabular}{|c|c|c|c|}
\hline$n$ & $\lambda_{n, s l 2}$ & TOL & IFLAG \\
\hline 0 & $-0.157663483 D+00$ & $0.23950 D-13$ & 1 \\
\hline 1 & $0.209076065 D+01$ & $0.19238 D-13$ & 1 \\
\hline 2 & $0.602403235 D+01$ & $0.20718 D-08$ & 1 \\
\hline 3 & $0.120111226 D+02$ & $0.10472 D-12$ & 1 \\
\hline 4 & $0.200064954 D+02$ & $0.70433 D-13$ & 1 \\
\hline
\end{tabular}

In all of the examples presented in this section we apply the FD-method with a uniform mesh on interval $[-1,1] ; N$ denotes the number of subintervals. The results obtained by the FD-method are presented in Tables $2-4$ and Figure 1 below. The results obtained by the SLEIGN2 are presented in Table 1 .

Analyzing the data in Tables 2, 3] and 4 we can conclude that the FD-method with $N=1$ converges much slower than the FD-method with $N=3$. This fact is in good agreement with the results of Theorem 2.1. Furthermore, as follows from Table 4 the convergence rate of the FD-method increases as the index $n$ of the trial eigenvalue increases. Figure1illustrates the exponential nature of the FD-method's convergence.
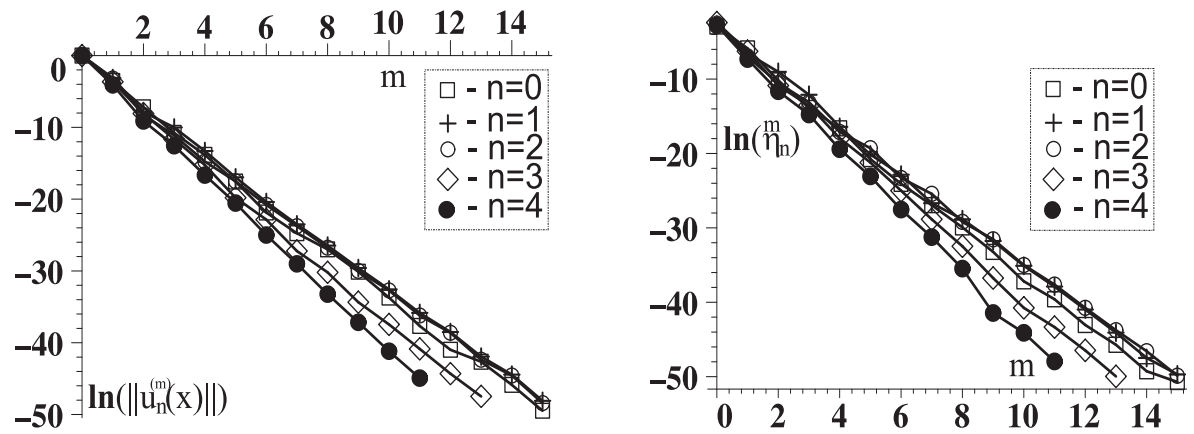

Figure 1. Example 1. The graphs of the functions $\ln \left(\left\|u_{n}^{(m)}(x)\right\|\right)$ (left) and $\ln \left(\stackrel{m}{\eta}_{n}\right)$ (right); $N=3$.

Example 2. As the second example, let us consider problem (4.1) with

$$
q(x)=\ln \left(\left|\left(\frac{5}{12}-x\right)\left(\frac{1}{3}+x\right)\right|\right) .
$$

The results obtained with SLEIGN2 are presented in Table 5 below.

It is worth emphasizing that the problem under consideration does not satisfy the conditions of Theorem 2.1. However, the results presented in Table 6 and Figure 2 show that the FD-method has successfully handled this problem. This is unlike to SLEIGN2 which gives results with substantial errors (see the leftmost colon in Table 6). 
TABLE 2. Example 1. The 0 -th eigenvalue, FD-method with $N=1$

\begin{tabular}{|c|c|c|c|c|}
\hline$m$ & $\dot{\lambda}_{0}$ & $\left\|u_{0}^{(m)}(x)\right\|$ & $\bar{\eta}_{n}$ & $\left|\begin{array}{|}m \\
\lambda_{0}\end{array} \lambda_{0, s l 2}\right|$ \\
\hline 0 & 0.0 & 1.0 & 0.56 & 0.157663483 \\
\hline 1 & 0.0 & 0.29 & 0.22 & 0.157663483 \\
\hline 2 & -0.1666666667 & $0.24 e-1$ & $0.36 \times 10^{-1}$ & $0.90031875 \times 10^{-2}$ \\
\hline 3 & -0.1666666667 & $0.18 \times 10^{-1}$ & $0.16 \times 10^{-1}$ & $0.90031875 \times 10^{-2}$ \\
\hline 4 & -0.1564814815 & $0.21 \times 10^{-2}$ & $0.48 \times 10^{-2}$ & $0.11819977 \times 10^{-2}$ \\
\hline 5 & -0.1564814815 & $0.24 \times 10^{-2}$ & $0.23 \times 10^{-2}$ & $0.11819977 \times 10^{-2}$ \\
\hline 6 & -0.1578630218 & $0.30 \times 10^{-3}$ & $0.78 \times 10^{-3}$ & $0.1995426 \times 10^{-3}$ \\
\hline 7 & -0.1578630218 & $0.41 \times 10^{-3}$ & $0.41 \times 10^{-3}$ & $0.1995426 \times 10^{-3}$ \\
\hline 8 & -0.1576253633 & $0.52 \times 10^{-4}$ & $0.15 \times 10^{-3}$ & $0.381159 \times 10^{-4}$ \\
\hline 9 & -0.1576253633 & $0.79 \times 10^{-4}$ & $0.78 \times 10^{-4}$ & $0.381159 \times 10^{-4}$ \\
\hline 10 & -0.1576713252 & $0.10 \times 10^{-4}$ & $0.31 \times 10^{-4}$ & $0.78460 \times 10^{-5}$ \\
\hline
\end{tabular}

TABLE 3. Example 1. The 0-th eigenvalue, FD-method with $N=1$

\begin{tabular}{|c|c|c|c|c|}
\hline$m$ & $\lambda_{0}$ & $\left\|u_{0}^{(m)}(x)\right\|$ & $\bar{m}_{n}$ & $\left|\begin{array}{l}m \\
\lambda_{0}\end{array} \lambda_{0, s l 2}\right|$ \\
\hline 51 & -0.15766348313775096746 & $0.12 \times 10^{-16}$ & $0.13 \times 10^{-16}$ & $0.39 \times 10^{-8}$ \\
\hline 52 & -0.15766348313775096031 & $0.16 \times 10^{-17}$ & $0.59 \times 10^{-17}$ & $0.39 \times 10^{-8}$ \\
\hline 53 & -0.15766348313775096031 & $0.33 \times 10^{-17}$ & $0.33 \times 10^{-17}$ & $0.39 \times 10^{-8}$ \\
\hline 54 & -0.15766348313775096218 & $0.39 \times 10^{-18}$ & $0.15 \times 10^{-17}$ & $0.39 \times 10^{-8}$ \\
\hline 55 & -0.15766348313775096218 & $0.84 \times 10^{-18}$ & $0.86 \times 10^{-18}$ & $0.39 \times 10^{-8}$ \\
\hline 56 & -0.15766348313775096169 & $0.11 \times 10^{-18}$ & $0.40 \times 10^{-18}$ & $0.39 \times 10^{-8}$ \\
\hline 57 & -0.15766348313775096169 & $0.22 \times 10^{-18}$ & $0.22 \times 10^{-18}$ & $0.39 \times 10^{-8}$ \\
\hline 58 & -0.15766348313775096182 & $0.28 \times 10^{-19}$ & $0.11 \times 10^{-18}$ & $0.39 \times 10^{-8}$ \\
\hline 59 & -0.15766348313775096182 & $0.58 \times 10^{-19}$ & $0.61 \times 10^{-19}$ & $0.39 \times 10^{-8}$ \\
\hline 60 & -0.15766348313775096178 & $0.74 \times 10^{-20}$ & $0.29 \times 10^{-19}$ & $0.39 \times 10^{-8}$ \\
\hline
\end{tabular}

TABLE 4. Example 1. FD-method with $N=3$

\begin{tabular}{|c|c|c|c|c|c|c|}
\hline$n$ & $m$ & $m$ & $\frac{\left|\lambda_{n}^{(m)}\right|}{10^{-20}}$ & $\frac{\left\|u_{n}^{(m)}(x)\right\|}{10^{-20}}$ & $\frac{\eta_{n}^{m}}{10^{-21}}$ & $\left|\begin{array}{l}m \\
\lambda_{n}\end{array}-\lambda_{n, s l 2}\right|$ \\
\hline 0 & 15 & -0.1576634831377509617898 & 0.03 & 0.033 & 0.095 & $0.39 \times 10^{-8}$ \\
\hline 1 & 15 & 2.090760648363956948786 & 0.7 & 0.13 & 0.26 & $0.1482 \times 10^{-5}$ \\
\hline 2 & 15 & 6.024031655336352711291 & 0.7 & 0.094 & 0.22 & $0.5035 \times 10^{-5}$ \\
\hline 3 & 13 & 12.01112256362987127625 & 1 & 0.24 & 0.20 & $0.4 \times 10^{-7}$ \\
\hline 4 & 11 & 20.00649533292656299628 & 1 & 3.1 & 1.5 & $0.7 \times 10^{-7}$ \\
\hline
\end{tabular}

TABLE 5. Example 2. The data obtained by SLEIGN2

\begin{tabular}{|c|c|c|c|}
\hline$n$ & $\lambda_{n, s l 2}$ & TOL & IFLAG \\
\hline 0 & $-1.98326983 D+00$ & $0.46748 D-08$ & 1 \\
\hline 1 & $0.855187683 D+00$ & $0.73426 D-07$ & 1 \\
\hline 2 & $0.489606686 D+01$ & $0.35447 D-07$ & 1 \\
\hline 3 & $0.104183770 D+02$ & $0.40228 D-07$ & 1 \\
\hline 4 & $0.188163965 D+02$ & $0.61329 D-11$ & 1 \\
\hline
\end{tabular}


TABle 6. Example 2. FD-method with $N=24$

\begin{tabular}{|c|c|c|c|c|c|c|}
\hline$n$ & $m$ & $\lambda_{n}$ & $\frac{\left|\lambda_{n}^{(m)}\right|}{10^{-16}}$ & $\frac{\left\|u_{n}^{(m)}(x)\right\|}{10^{-17}}$ & $\frac{\eta_{n}^{m}}{10^{-17}}$ & $\left|\lambda_{n}^{m}-\lambda_{n, s l 2}\right|$ \\
\hline 0 & 8 & -1.9831442709774408386 & 0.099 & 0.26 & 0.02 & $0.12556 \times 10^{-3}$ \\
\hline 1 & 8 & 0.85727032837311800023 & 4.7 & 7.3 & 0.44 & $0.2082645 \times 10^{-2}$ \\
\hline 2 & 8 & 4.8939506826799075597 & 0.098 & 0.29 & 0.051 & $0.211618 \times 10^{-2}$ \\
\hline 3 & 8 & 10.4205112962574333545 & 0.03 & 7.4 & 1.3 & $0.213430 \times 10^{-2}$ \\
\hline 4 & 7 & 18.816396521508987920 & 0.11 & 0.87 & 0.039 & $0.2 \times 10^{-7}$ \\
\hline
\end{tabular}
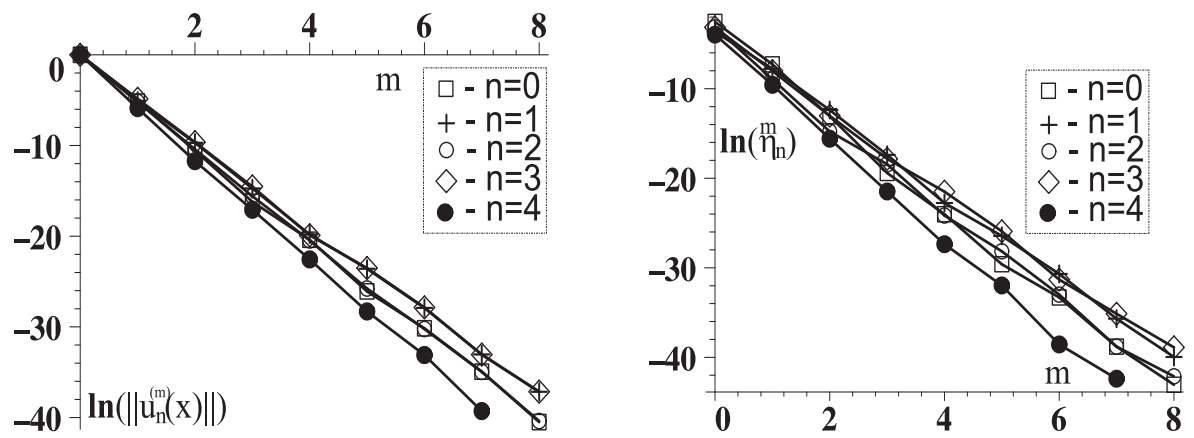

Figure 2. Example 2. The graphs of the functions $\ln \left(\left\|u_{n}^{(m)}(x)\right\|\right)$ (left) and $\ln \left(\stackrel{m}{\eta}_{n}\right)$ (right); $N=24$.

Example 3. Finally, let us consider problem (4.1) with

$$
q(x)=\frac{1}{\sqrt{\left|x+\frac{1}{3}\right|}}+\ln \left(\left|x-\frac{1}{3}\right|\right) .
$$

We find out that SLEIGN2 cannot handle this problem while the FD-method can. The results obtained with the FD-method are presented in Table 7 and Figure 3. As before, Figure 3 confirms the exponential convergence rate of the method.
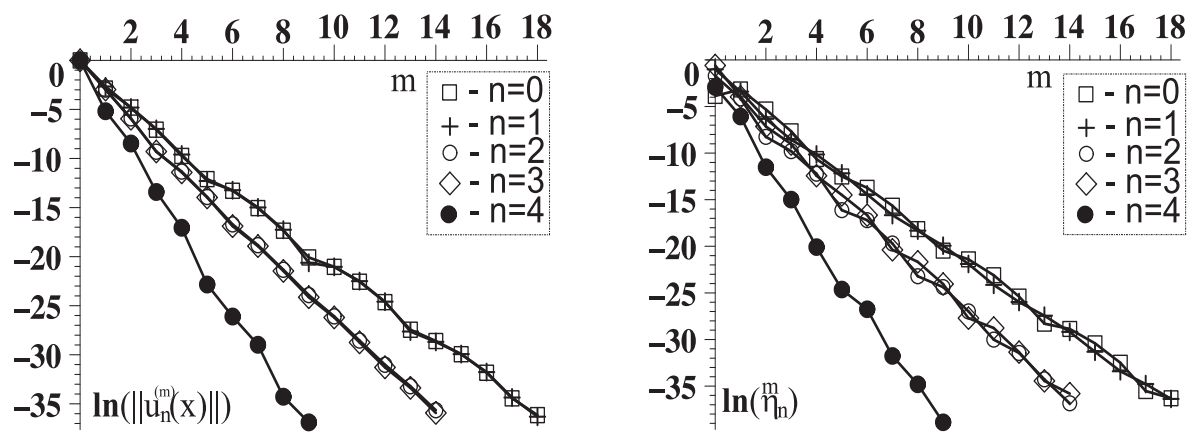

Figure 3. Example 3. The graphs of the functions $\ln \left(\left\|u_{n}^{(m)}(x)\right\|\right)$ (left) and $\ln \left(\stackrel{m}{\eta}_{n}\right)$ (right); $N=12$. 
TABle 7. Example 3. FD-method with $N=12$

\begin{tabular}{|c|c|c|c|c|c|}
\hline$n$ & $m$ & $m_{n}$ & $\left|\lambda_{n}^{(m)}\right|$ & $\left\|u_{n}^{(m)}(x)\right\|$ & $\stackrel{m}{\eta}_{n}$ \\
\hline 0 & 18 & 0.40796999146419634 & $0.42 \times 10^{-15}$ & $0.20 \times 10^{-15}$ & $0.17 \times 10^{-15}$ \\
\hline 1 & 18 & 3.4136861164474333 & $0.4 \times 10^{-15}$ & $0.17 \times 10^{-15}$ & $0.16 \times 10^{-15}$ \\
\hline 2 & 14 & 6.7759537951814352 & $0.2 \times 10^{-15}$ & $0.33 \times 10^{-15}$ & $0.10 \times 10^{-15}$ \\
\hline 3 & 14 & 13.323487340142488 & $0.2 \times 10^{-14}$ & $0.25 \times 10^{-15}$ & $0.28 \times 10^{-15}$ \\
\hline 4 & 9 & 20.8431972121837340 & $0.1 \times 10^{15}$ & $0.96 \times 10^{-16}$ & $0.13 \times 10^{-16}$ \\
\hline
\end{tabular}

\section{Conclusions}

In the present paper we have constructed and theoretically justified a generalized algorithm of the FD-method for solving the Sturm-Liouville problem for secondorder differential equation (1.1), (1.2) with piecewise continuous functional coefficient $q(x)$. As follows from Theorem 2.1, the generalized FD-method, which uses a piecewise constant approximation of the function $q(x)$, can be applied to approximate eigenvalues and eigenfunctions with any nonnegative index $n$. The convergence rate of the method can be increased by decreasing the value $\|q(x)-\bar{q}(x)\|_{\infty,[-1,1]}$. In the case when $\bar{q}(x) \equiv 0$ (this case was considered in [6]) the FD-method, if convergent, allows us to obtain the approximation for the eigensolution analytically. In general, however, when $\bar{q}(x) \neq 0$ the analytical approach fails and it is necessary to use numerical integration methods such as sinc quadratures and Stenger's formula (see [9, 12]).

The problems considered in Examples 2 and 3 do not satisfy the conditions of Theorem 1.1 because of the function $q(x)$ being unbounded on $[-1,1]$. However, as it has been shown in the examples above, the method successfully converges whereas the well-known and widely applied SLEIGN2 package either provides no more than three correct digits after decimal point (Example 2) or cannot handle the problem at all (Example 3). These examples indicate that the FD-method has considerable potential that is to be investigated in further mathematical works.

\section{REFERENCES}

1. P. B. Bailey, W. N. Everitt, and A. Zettl, Algorithm 810: The Sleign2 Sturm-Liouville code, ACM Trans. Math. Softw. 27 (2001), 143-192.

2. B. Curtis Eaves, Floyd J. Gould, Heinz-Otto Peitgen, and Michael J. Todd (eds.), Homotopy methods and global convergence, NATO Conference Series II: Systems Science, vol. 13, New York, Plenum Press, 1983. MR749963 (85h:90113)

3. I. P. Gavrilyuk, A. V. Klimenko, V. L. Makarov, and N. O. Rossokhata, Exponentially convergent parallel algorithm for nonlinear eigenvalue problems, IMA J. Numer. Anal. 27 (2007), no. 4, 818-838. MR2371834 (2008k:65151)

4. H. Bateman and A. Erdely, Vysshie transtsendentnye funktsii. I: Gipergeometricheskaya funktsiya. Funktsii Lezhandra, unrevised ed., Izdat. "Nauka", Moscow, 1973, Translated from the English by N. Ja. Vilenkin. MR0344526 (49:9265)

5. N. Kryloff and N. Bogolioubov, Sopra il metodo del coefficient constati (metodo del tronconi) per l'integrazione approssimate delle equazioni differenziali delle fisica mathematica, Boll. Unione Mat. Ital. (1926), no. 7, 72-76.

6. V. L. Makarov and Ya. V. Klimenko, Application of the functional-discrete method to the solution of the Sturm-Liouville problem with coefficients of a special form, Ukraïn. Mat. Zh. 59 (2007), no. 8, 1140-1147. MR2397707(2009b:65176) 
7. V.L. Makarov, About functional-discrete method of arbitrary accuracy order for solving Sturm-Liouville problem with piecewise smooth coefficients, Dokl. Akad. Nauk. SSSR 320 (1991), no. 1, 34-39. MR1151511 (93c:65099)

8. V.L. Makarov and N.O. Rossokhata, A review of functional-discrete technique for eigenvalue problems, J. Numer. Appl. Math. (2009), no. 97, 79-102, available at http://www.nbuv.gov.ua/portal/natural/jopm/2009_1/index.htm.

9. Stephen Nash, David Kahaner, and Clev Moler, Numerical methods and software, PrenticeHall, Inc., New Jersey, 1989, 495 pp.

10. J.D. Pryce, Numerical solution of Sturm-Liouville problems, Clarendon Press, Oxford, New York, Tokyo, 1993, 322 pp. MR.1283388 (95h:65056)

11. Sergei Yu. Slavyanov and Wolfgang Lay, Special functions, Oxford Mathematical Monographs, Oxford University Press, Oxford, 2000, A unified theory based on singularities, With a foreword by Alfred Seeger, Oxford Science Publications. MR.1858237 (2004e:33003)

12. Frank Stenger, Numerical methods based on sinc and analytic functions, Springer Series in Computational Mathematics, vol. 20, Springer-Verlag, New York, 1993. MR.1226236 (94k:65003)

Department of Numerical Mathematics, Institute of Mathematics of NAS of Ukraine,

3 Tereshchenkivs'Ka Str., Kyiv-4, 01601, Ukraine

E-mail address: makarov@imath.kiev.ua

Department of Numerical Mathematics, Institute of Mathematics of NAS of Ukraine, 3 Tereshchenkivs'Ka Str., Kyiv-4, 01601, Ukraine

E-mail address: dragunovdenis@gmail.com

Department of Numerical Mathematics, Institute of Mathematics of NAS of Ukraine,

3 Tereshchenkivs'Ka Str., Kyiv-4, 01601, Ukraine

E-mail address: oldyara@gmail.com 